\title{
Dynamic Behavior for an SIRS Model with Nonlinear Incidence Rate and Treatment
}

\author{
Junhong Li and Ning Cui \\ Department of Mathematics and Sciences, Hebei Institute of Architecture \& Civil Engineering, Zhangjiakou, Hebei 075000, China
}

Correspondence should be addressed to Junhong Li; jhli2011@163.com

Received 23 September 2013; Accepted 6 November 2013

Academic Editors: W. Jarczyk and L. Kocinac

Copyright (C) $2013 \mathrm{~J}$. Li and N. Cui. This is an open access article distributed under the Creative Commons Attribution License, which permits unrestricted use, distribution, and reproduction in any medium, provided the original work is properly cited.

This paper considers an SIRS model with nonlinear incidence rate and treatment. It is assumed that susceptible and infectious individuals have constant immigration rates. We investigate the existence of equilibrium and prove the global asymptotical stable results of the endemic equilibrium. We then obtained that the model undergoes a Hopf bifurcation and existences a limit cycle. Some numerical simulations are given to illustrate the analytical results.

\section{Introduction}

Treatment is an important and effective method to prevent and control the spread of various infectious diseases, such as measles, tuberculosis, and flu [1-4]. In classical epidemic models, the treatment rate of the infective is assumed to be proportional to the number of the infective individuals [5]. This is unsatisfactory because the resources for treatment should be quite large. In fact, every community should have a suitable capacity for treatment. If it is too large, the community pays for unnecessary cost. If it is small, the community has the risk of the outbreak of a disease. Thus, it is realistic to maintain a suitable capacity of disease treatment. Wang and Ruan [6] considered an SIR model in which the capacity for the treatment of a disease in a community is a constant. Namely, they used the following function:

$$
h(I)= \begin{cases}k, & \text { if } I>0, \\ 0, & \text { if } I=0,\end{cases}
$$

which was used by [7]. This seems more reasonable when we consider the limitation of the treatment resource of a community.

There are many reasons for using nonlinear incidence rate, and various forms of nonlinear incidence rates have been proposed recently. Liu et al. [8] proposed a nonlinear saturated incidence function $g(I) S=\beta S I^{p} /\left(1+\alpha I^{q}\right)$ to model the effect of behavioral changes to certain communicable disease, where $\beta S I^{p}$ describes the infection force of the disease and $1 /\left(1+\alpha I^{q}\right)$ measures the inhibition effect from the behavioral change of the susceptible individuals when the number of infectious individuals increases. The case when $p=1, q=2$ was used by [9]. We assume the population can be partitioned into three compartments: susceptible, infective, and recovered. Let $S, I$, and $R$ denote the numbers of susceptible, infective, and recovered individuals, respectively. Motivated by the works $[6,7,9]$, we will formulate an SIRS model with nonlinear incidence rate and constant immigration rates for susceptible and infectious individuals [10]. Namely, we consider the following SIRS model:

$$
\begin{gathered}
S^{\prime}=(1-p) A-\frac{\beta I S}{1+\alpha I^{2}}-d S+\gamma R, \\
R^{\prime}=m I-(d+\gamma) R+h(I), \\
I^{\prime}=p A+\frac{\beta I S}{1+\alpha I^{2}}-(d+m) I-h(I),
\end{gathered}
$$

where $d$ is the rate of natural death, $m$ is the rate for recovery, $\beta$ is the proportionality constant, $\gamma$ is the rate at which recovered individuals lose immunity and return to susceptible class, $\alpha$ is the parameter measures of the psychological or inhibitory effect, and $(1-p) A, p A$ are constant recruitments of susceptible and infective individuals, respectively. It is assumed that all the parameters are positive constants. It is easy to see that the total population size $N$ implies 
$N^{\prime}=A-d N$. Since $N$ tends to a constant as $t$ tends to infinity, we assume that the population is in equilibrium and investigate the behavior of (2) on the plane $S+I+R=A / d=$ $N_{0}>0$. Let $(S(t), I(t), R(t))$ be a solution of (2) with initial conditions $S(0) \geq 0, I(0) \geq 0$, and $R(0) \geq 0$. This solution will satisfy $S(t) \geq 0, I(t) \geq 0$, and $R(t) \geq 0$ for all $t \geq 0$ since $S^{\prime}=(1-p) A+\gamma R>0$ if $(0, I(t), R(t)) \in R_{+}^{3}, I^{\prime}=p A>0$ if $(S(t), 0, R(t)) \in R_{+}^{3}$, and $R^{\prime}=m I+h(I) \geq 0$ if $(S(t), I(t), 0) \epsilon$ $R_{+}^{3}$. Consequently, $R_{+}^{3}$ is positively invariant for (2). Thus, we restrict our attention to the following reduced model:

$$
\begin{gathered}
I^{\prime}=p A-k+\frac{\beta I\left(N_{0}-I-R\right)}{1+\alpha I^{2}}-(d+m) I, \\
R^{\prime}=m I-(d+\gamma) R+k,
\end{gathered}
$$

where $k$ is the treatment constant.

From the epidemiological interpretation, our discussion on (3) will be restricted in the following bounded domain:

$$
D=\left\{(I, R): I>0, R>0,0<I+R<N_{0}\right\}
$$

which is a positively invariant set for (3).

The paper is organized as follows. In the next section, we investigate the existence and stability of equilibrium for (3). In Section 3, we study the Hopf bifurcation and limit cycle. Some numerical simulations are given to illustrate the analytical results. Section 4 is a brief discussion.

\section{Existence and Stability of Equilibrium}

In this section, we first consider the existence of equilibrium of (3) and their global stability. In order to find endemic equilibrium of (3), we substitute

$$
R=\frac{m I+k}{d+\gamma}
$$

into

$$
p A-k+\frac{\beta I\left(N_{0}-I-R\right)}{1+\alpha I^{2}}=(d+m) I
$$

to obtain the cubic equation

$$
\begin{aligned}
f(I)= & \alpha(d+m) I^{3}+\left[\frac{\beta(d+\gamma+m)}{d+\gamma}+(k-p A) \alpha\right] I^{2} \\
& +\left[d+m+\frac{\beta k}{d+\gamma}-\beta N_{0}\right] I+k-p A=0 .
\end{aligned}
$$

Let $I_{1}, I_{2}$, and $I_{3}$ be three roots of (7). Then, we get

$$
\begin{aligned}
& \frac{\beta}{\alpha(d+m)}\left(\frac{A}{d}-\frac{k}{d+\gamma}\right)=\frac{1}{\alpha}-\left(I_{1} I_{2}+I_{1} I_{3}+I_{3} I_{2}\right), \\
& \frac{\beta}{\alpha(d+m)}\left(1+\frac{m}{d+\gamma}\right)=I_{1} I_{2} I_{3} \alpha-\left(I_{1}+I_{2}+I_{3}\right) .
\end{aligned}
$$

When $R_{0}=k / p A<1$, we can see that

$$
\frac{I_{1}+I_{2}+I_{3}}{I_{1} I_{2} I_{3}}<\alpha<\frac{1}{I_{1} I_{2}+I_{2} I_{3}+I_{1} I_{3}}
$$

then there is a unique positive root of (7). Direct calculations show that

$$
\begin{aligned}
f\left(N_{0}\right)= & (1-p) B+\gamma N_{0} \\
+ & N_{0}^{2}\left[\frac{\beta \gamma N_{0}}{(d+\varepsilon)}+\gamma \alpha N_{0}+(1-p) d \alpha N_{0}\right]>0, \\
f\left(\frac{p A-k}{d+m}\right)= & \frac{\beta p A\left(1-R_{0}\right)}{(d+m)^{2}(d+\gamma)} \\
& \times\left[-k \gamma+N_{0}(p-1) d(d+\gamma+m)-N_{0} \gamma m\right] \\
& <0 \quad \text { if } R_{0}<1 .
\end{aligned}
$$

From biological considerations, it is easy to see the positive root

$$
I \in\left(\frac{p A-k}{d+m}, N_{0}\right)
$$

Based on the above analysis, we obtain the following theorem.

Theorem 1. There is a unique endemic equilibrium $E_{0}\left(I_{e}, R_{e}\right)$ of (3) if $R_{0}<1$.

Theorem 2. The endemic equilibrium $E_{0}$ of (3) is locally asymptotically stable if $R_{0}<1$.

Proof. The Jacobian matrix of (3) at $E_{0}$ takes the form

$$
\begin{aligned}
& J\left(E_{0}\right)= \\
& \qquad\left[\begin{array}{cc}
\frac{p A\left(R_{0}-1\right)}{I_{e}}-\frac{2 \alpha \beta I_{e}^{2}\left[N_{0}-I_{e}-R_{e}\right]}{\left(1+\alpha I_{e}^{2}\right)^{2}}-\frac{\beta I_{e}}{1+\alpha I_{e}^{2}} & -\frac{\beta I_{e}}{1+\alpha I_{e}^{2}} \begin{array}{c}
-d-\gamma \\
m
\end{array}
\end{array} .\right.
\end{aligned}
$$

It is easy to obtain $\operatorname{tr}\left(J\left(E_{0}\right)\right)<0$ and $\operatorname{det}\left(J\left(E_{0}\right)\right)>0$ when $R_{0}<1$. This completes the proof.

Theorem 3. The endemic equilibrium $E_{0}$ of (3) is globally asymptotically stable if $R_{0}<1$.

Proof. Taking Dulac function

$$
D=\frac{1+\alpha I^{2}}{I}
$$


we obtain

$$
\begin{aligned}
\frac{\partial(P D)}{\partial I}+\frac{\partial(Q D)}{\partial R}= & -\beta-2 \alpha(d+m) I-\frac{p A\left(1-R_{0}\right)}{I^{2}} \\
& +\alpha p A\left(1-R_{0}\right)-\frac{d+\gamma}{I}-\alpha I(d+\gamma) \\
\leq & -\beta-\alpha p A\left(1-R_{0}\right) I \\
& -\frac{p A\left(1-R_{0}\right)}{I^{2}}-\frac{d+\gamma}{I}-\alpha I(d+\gamma),
\end{aligned}
$$

where $(P, Q)$ is the vector field of (3). Obviously,

$$
\frac{\partial(P D)}{\partial I}+\frac{\partial(Q D)}{\partial R}<0 \quad \text { if } R_{0}<1
$$

Then by Dulac's criteria, the system (3) admits no limit cycles or separatrix cycles. The global stability of $E_{0}$ follows from the Poincare-Bendixson Theorem. This completes the proof.

Theorem 4. There will be one or two endemic equilibria $E_{*}\left(I_{*}, R_{*}\right)$ of the system (3) if $R_{0}>1$.

Proof. Based on the analysis of Theorem 1, when $R_{0}>1$, we obtain the roots of (7) satisfying

$$
\begin{gathered}
I_{1}+I_{2}+I_{3}=-\frac{\beta(d+\gamma+m)+\alpha p A\left(R_{0}-1\right)(d+\gamma)}{\alpha(d+m)(d+\gamma)}<0, \\
I_{1} I_{2} I_{3}=-\frac{p A\left(R_{0}-1\right)}{\alpha(d+m)}<0 ;
\end{gathered}
$$

then there exist one positive real root and two conjugate complex roots with negative real parts or two positive real roots and one negative root. Thus, there will be one or two endemic equilibria in (3). This completes the proof.

\section{Hopf Bifurcation}

In this section, we study the Hopf bifurcation and limit cycle of the system (3). For simplicity of computation, we consider the following system which is equivalent to (3):

$$
\begin{aligned}
I^{\prime}= & \beta I\left(N_{0}-I-R\right)-(d+m) I\left(1+\alpha I^{2}\right) \\
& +\left(1-R_{0}\right) p A\left(1+\alpha I^{2}\right), \\
R^{\prime}= & {\left[m I-(d+\gamma) R+p A R_{0}\right]\left(1+\alpha I^{2}\right) . }
\end{aligned}
$$

Let $x=I-I_{*}, y=R-R_{*}$ to translate $E_{*}$ to $(0,0)$. Then, (17) becomes

$$
\begin{aligned}
& x^{\prime}=a_{11} x+a_{12} y+f_{1}(x, y), \\
& y^{\prime}=a_{21} x+a_{22} y+f_{2}(x, y),
\end{aligned}
$$

where $f_{1}(x, y)$ and $f_{2}(x, y)$ represent the higher order terms and

$$
\begin{gathered}
a_{11}=\beta\left(N_{0}-2 I_{*}-R_{*}\right)-(d+m)\left(1+3 \alpha I_{*}^{2}\right) \\
-2 p A \alpha\left(R_{0}-1\right) I_{*}, \\
a_{12}=-\beta I_{*}, \quad a_{21}=m\left(1+\alpha I_{*}^{2}\right), \\
a_{22}=-(d+\gamma)\left(1+\alpha I_{*}^{2}\right) .
\end{gathered}
$$

To obtain the Hopf bifurcation, we fix parameters such that $\operatorname{tr}\left(J\left(E_{*}\right)\right)=0$, which is equivalent to $a_{11}=(d+\gamma)\left(1+\alpha I_{*}^{2}\right)$. Let

$$
X=x, \quad Y=a_{11} x+a_{12} y
$$

then (18) is reduced to

$$
\begin{gathered}
X^{\prime}=Y+f_{1}\left(X, \frac{Y-a_{11} X}{a_{12}}\right), \\
Y^{\prime}=-\delta X+a_{11} f_{1}\left(X, \frac{Y-a_{11} X}{a_{12}}\right) \\
+a_{12} f_{2}\left(X, \frac{Y-a_{11} X}{a_{12}}\right),
\end{gathered}
$$

where

$$
\begin{gathered}
\delta=(d+r)^{2}\left(1+\alpha I_{*}^{2}\right)^{2}-m \beta I_{*}\left(1+\alpha I_{*}^{2}\right)>0 \\
\text { if }(d+r)^{2}\left(1+\alpha I_{*}^{2}\right)>m \beta I_{*} .
\end{gathered}
$$

Let

$$
u=-X, \quad v=\frac{Y}{\sqrt{\delta}}
$$

we obtain the normal form of the Hopf bifurcation:

$$
\begin{aligned}
& u^{\prime}=-\sqrt{\delta} v+F_{1}(u, v), \\
& v^{\prime}=\sqrt{\delta} u+F_{2}(u, v),
\end{aligned}
$$

where

$$
\begin{gathered}
F_{1}(u, v)=\frac{\sqrt{\delta} u}{I_{*}}-(d+m) \alpha u^{3} \\
-\left[\beta+3 d \alpha I_{*}+3 m \alpha I_{*}-p A \alpha\left(R_{0}-1\right)\right. \\
\left.-\frac{(d+\alpha)\left(1+\alpha I_{*}^{2}\right)}{I_{*}}\right] u^{2},
\end{gathered}
$$




$$
\begin{aligned}
& F_{2}(u, v) \\
& =\frac{1}{\sqrt{\delta}}\left[-m \alpha+\frac{\alpha(d+\gamma)^{2}\left(1+\alpha I_{*}^{2}\right)}{\beta I_{*}}\right] u^{3} \\
& \quad+\frac{1}{\sqrt{\delta}}\left[2 m \alpha I_{*}-\frac{2 \alpha(d+\gamma)^{2}\left(1+\alpha I_{*}^{2}\right)}{\beta}\right] \\
& \quad+\frac{\alpha(d+\gamma) u^{2} v}{\beta I_{*}}-\frac{m\left(1+\alpha I_{*}^{2}\right)}{\sqrt{\delta}} \\
& \quad-\frac{2 \alpha(d+\gamma) u v^{2}}{\beta}+\frac{(d+\gamma)\left(1+\alpha I_{*}^{2}\right) v}{\sqrt{\delta} \beta I_{*}} .
\end{aligned}
$$

Set the Lyapunov number by

$$
\begin{aligned}
\sigma=\frac{1}{16}[ & \frac{\partial^{3} F_{1}}{\partial u^{3}}+\frac{\partial^{3} F_{1}}{\partial u \partial v^{2}}+\frac{\partial^{3} F_{2}}{\partial u^{2} \partial v}+\frac{\partial^{3} F_{2}}{\partial v^{3}} \\
& +\frac{\partial^{2} F_{1}}{\partial u \partial v}\left(\frac{\partial^{2} F_{1}}{\partial u^{2}}+\frac{\partial^{2} F_{1}}{\partial v^{2}}\right)-\frac{\partial^{2} F_{2}}{\partial u \partial v}\left(\frac{\partial^{2} F_{2}}{\partial u^{2}}+\frac{\partial^{2} F_{2}}{\partial v^{2}}\right) \\
& \left.-\frac{\partial^{2} F_{1}}{\partial u^{2}} \frac{\partial^{2} F_{2}}{\partial u^{2}}+\frac{\partial^{2} F_{1}}{\partial v^{2}} \frac{\partial^{2} F_{2}}{\partial v^{2}}\right],
\end{aligned}
$$

which can be reduced to

$$
\sigma=\frac{1}{16}\left[\frac{2 \alpha(d+\gamma)}{\beta I_{*}}-6 \alpha(d+m)\right] .
$$

So we have the following Hopf bifurcation results.

Theorem 5. There exist Hopf bifurcation and limit cycle in the system (17), when

$$
a_{11}=(d+\gamma)\left(1+\alpha I_{*}^{2}\right), \quad(d+r)^{2}\left(1+\alpha I_{*}^{2}\right)>m \beta I_{*} .
$$

To illustrate the theorem, let us consider the following parameters.

$\beta=0.01$ (see [11]), $m=0.1$ (see [12]), $A=4.4236, d=$ $k=\gamma=0.1, \alpha=0.3995$, and $p=0.01$.

We have $R_{0}=2.2606>1$, and the equilibria $E_{1}(0.25$, $0.625), E_{2}(1.3505,1.1752)$ exist (see Theorem 4$)$. The equilibrium $E_{2}$ is unstable saddle. The parameter values satisfy conditions (29) of Theorem 5 and $\sigma=3.965$. Therefore, (17) has an unstable periodic orbit which encircles $E_{1}$. Its phase portrait is illustrated in Figure 1. The time series of the infective and recovered individuals are given in Figures 2 and 3 , respectively.

\section{Conclusion}

In this paper, we discuss an SIRS epidemic model with nonlinear incidence rate and treatment. It is assumed that susceptible and infectious individuals have constant immigration

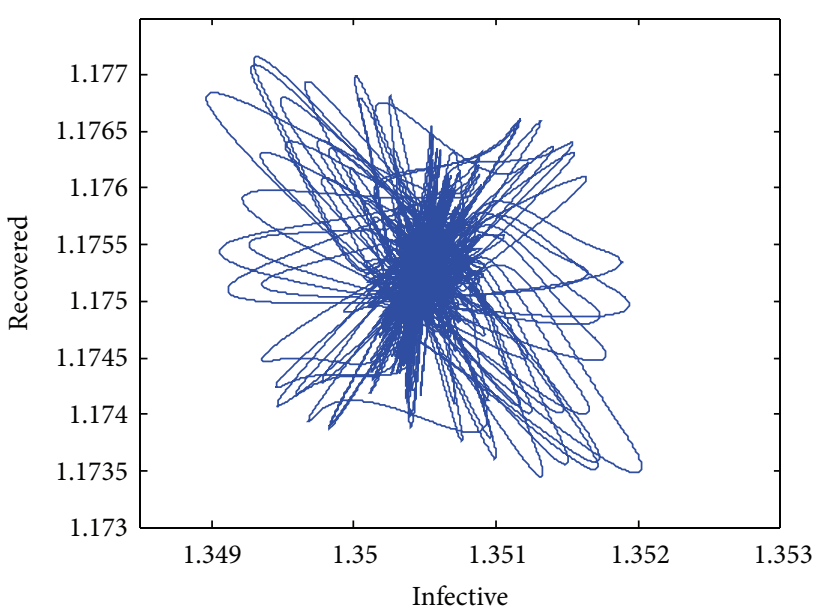

FIGURE 1: The phase portraits of (17).

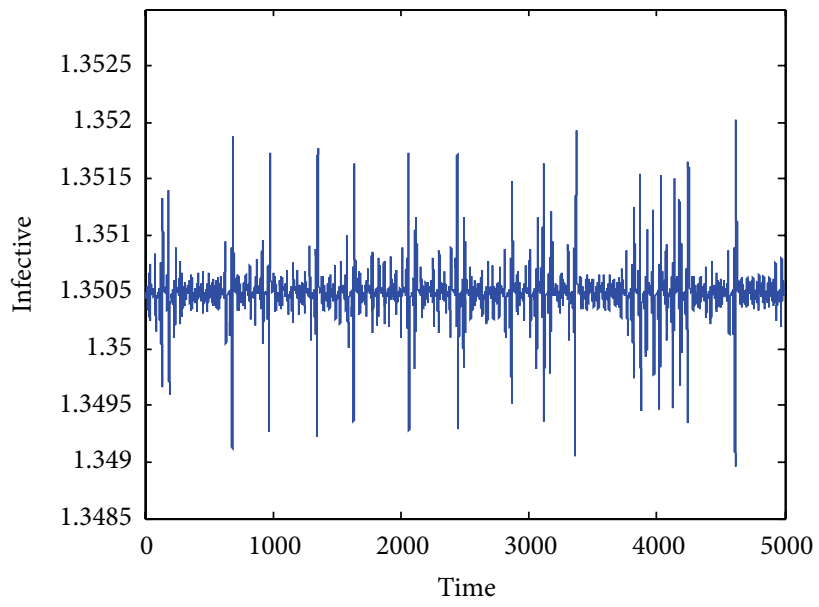

FIGURE 2: Time series of infective individuals.

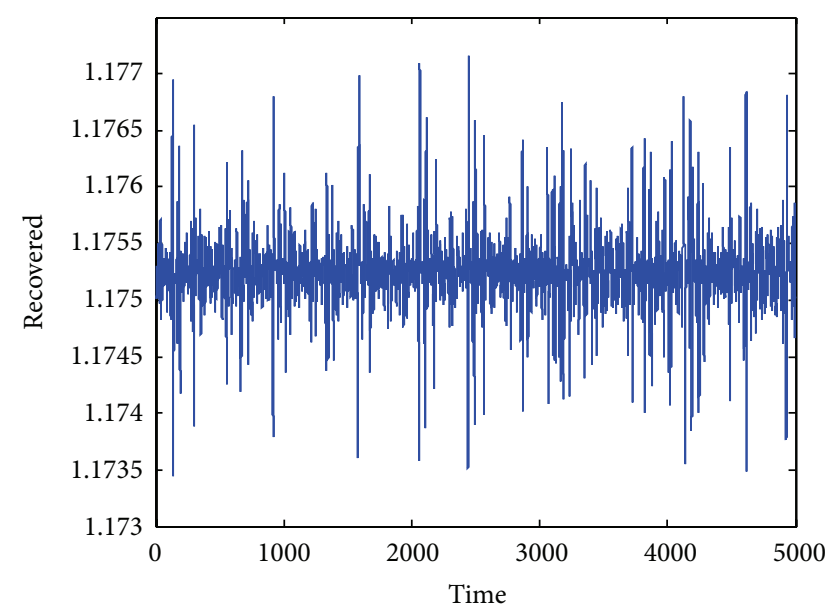

FIgURE 3: Time series of recovered individuals. 
rates. We investigate the existence and stability of equilibria of (3) and study the Hopf bifurcation and limit cycle. Some numerical simulations are given to illustrate the analytical results. Without the treatment and recruitment of infectious, (2) becomes the SIRS model (see [9]).

\section{Acknowledgments}

The authors are very grateful to the reviewers for their valuable comments and suggestions. This work was supported by the Youth Science Foundations of Education Department of Hebei Province (nos. 2010233 and 2011236).

\section{References}

[1] Z. Feng and H. R. Thieme, "Recurrent outbreaks of childhood diseases revisited: the impact of isolation," Mathematical Biosciences, vol. 128, no. 1-2, pp. 93-130, 1995.

[2] J. M. Hyman and J. Li, "Modeling the effectiveness of isolation strategies in preventing STD epidemics," SIAM Journal on Applied Mathematics, vol. 58, no. 3, pp. 912-925, 1998.

[3] L.-I. Wu and Z. Feng, "Homoclinic bifurcation in an SIQR model for childhood diseases," Journal of Differential Equations, vol. 168, no. 1, pp. 150-167, 2000.

[4] Z. Qiu and Z. Feng, "Transmission dynamics of an influenza model with vaccination and antiviral treatment," Bulletin of Mathematical Biology, vol. 72, no. 1, pp. 1-33, 2010.

[5] R. M. Anderson and R. M. May, Infectious Diseases of Humans, Oxford University Press, London, UK, 1991.

[6] W. Wang and S. Ruan, "Bifurcations in an epidemic model with constant removal rate of the infectives," Journal of Mathematical Analysis and Applications, vol. 291, no. 2, pp. 775-793, 2004.

[7] Z. Bai and Y. Zhou, "Existence of two periodic solutions for a non-autonomous SIR epidemic model," Applied Mathematical Modelling, vol. 35, no. 1, pp. 382-391, 2011.

[8] W.-M. Liu, S. A. Levin, and Y. Iwasa, "Influence of nonlinear incidence rates upon the behavior of SIRS epidemiological models," Journal of Mathematical Biology, vol. 23, no. 2, pp. 187204, 1985.

[9] D. Xiao and S. Ruan, "Global analysis of an epidemic model with nonmonotone incidence rate," Mathematical Biosciences, vol. 208, no. 2, pp. 419-429, 2007.

[10] G. Li, W. Wang, and Z. Jin, "Global stability of an SEIR epidemic model with constant immigration," Chaos, Solitons and Fractals, vol. 30, no. 4, pp. 1012-1019, 2006.

[11] L. Zhou and M. Fan, "Dynamics of an SIR epidemic model with limited medical resources revisited," Nonlinear Analysis: Real World Applications, vol. 13, no. 1, pp. 312-324, 2012.

[12] X.-Z. Li, W.-S. Li, and M. Ghosh, "Stability and bifurcation of an SIR epidemic model with nonlinear incidence and treatment," Applied Mathematics and Computation, vol. 210, no. 1, pp. 141150, 2009. 


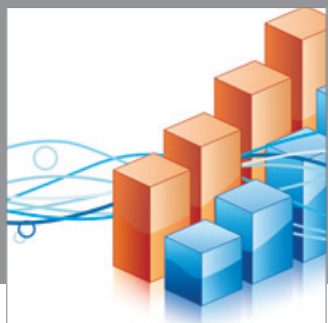

Advances in

Operations Research

mansans

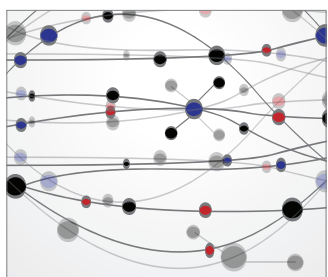

The Scientific World Journal
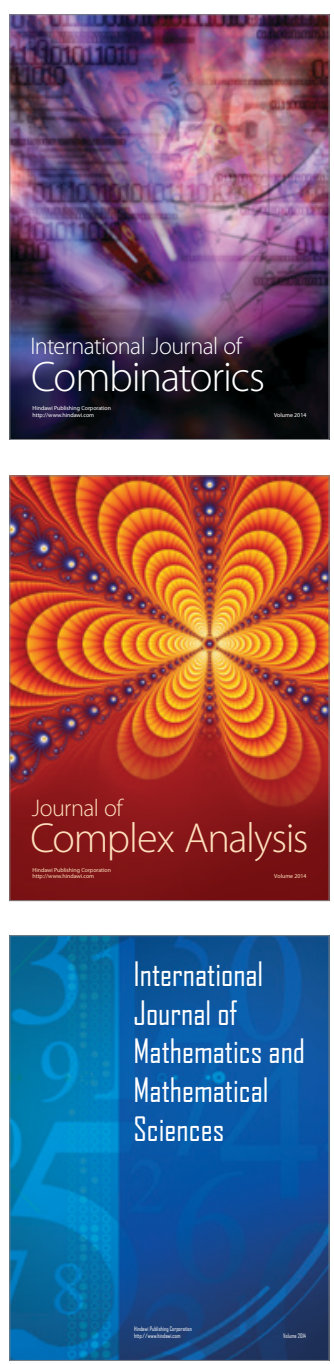
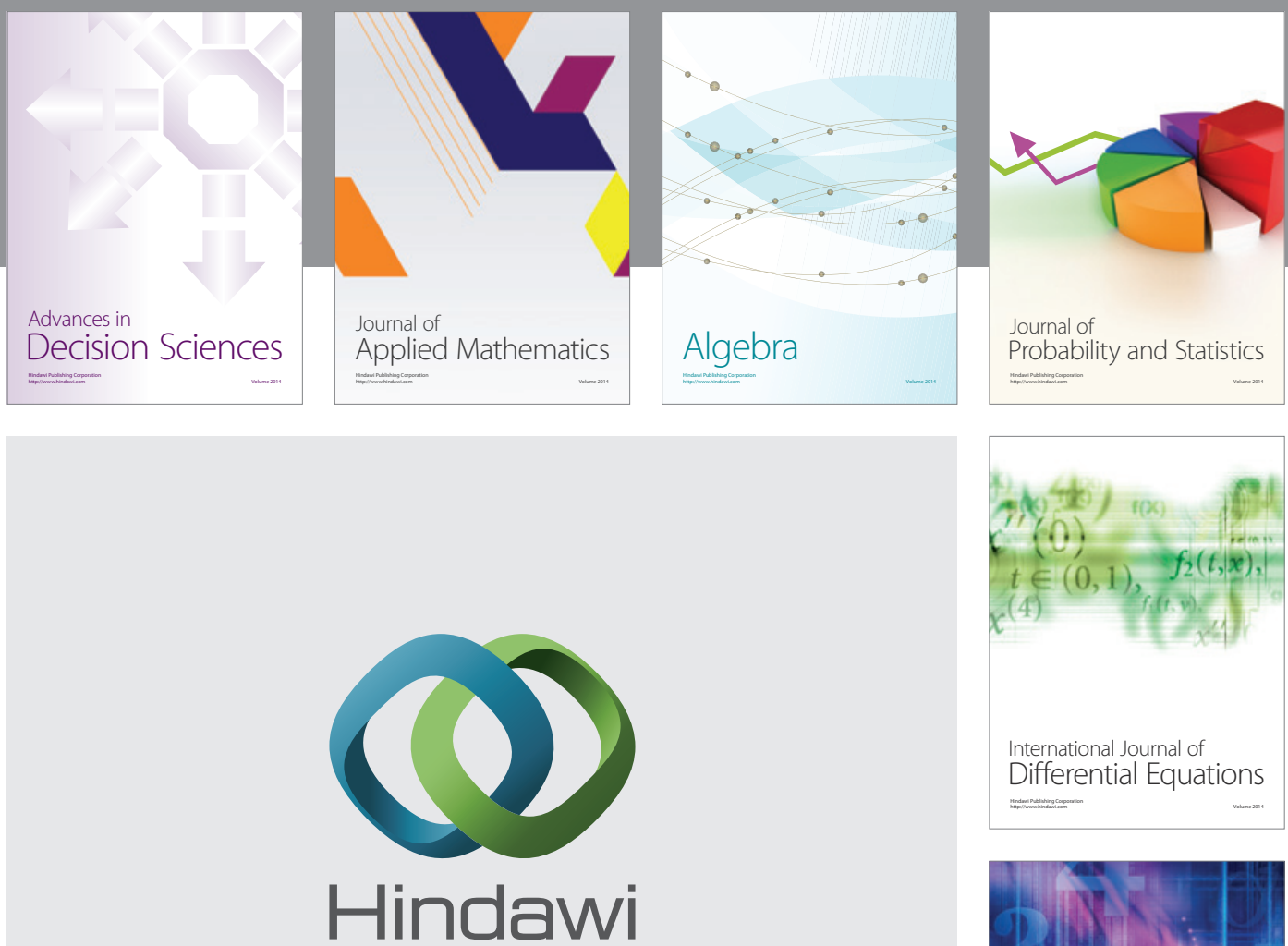

Submit your manuscripts at http://www.hindawi.com
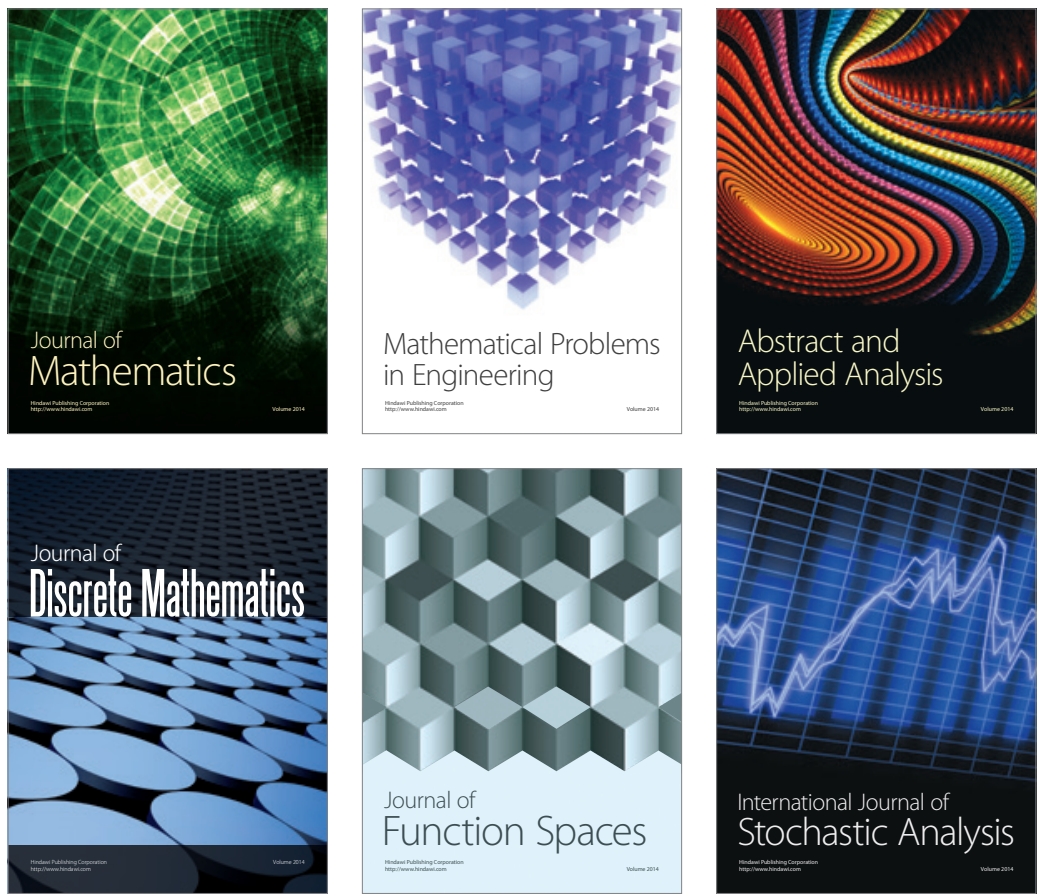

Journal of

Function Spaces

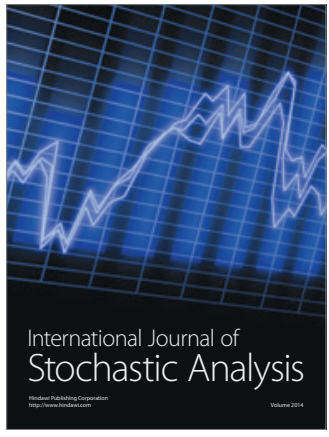

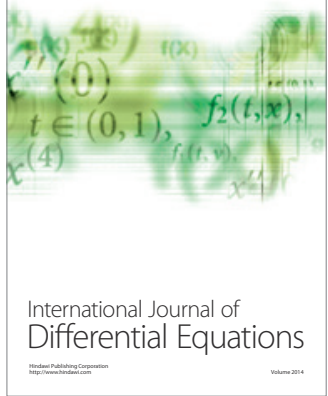
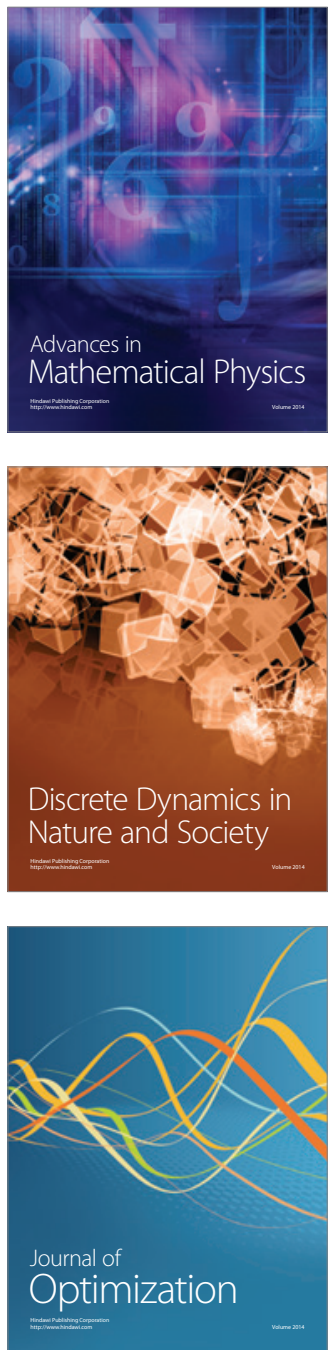